\title{
Tanshinone IIA reduces the risk of Alzheimer's disease by inhibiting iNOS, MMP-2 and NF-кBp65 transcription and translation in the temporal lobes of rat models of Alzheimer's disease
}

\author{
PING JIANG ${ }^{1}$, CHUNBO LI $^{1}$, ZHENGHUA XIANG $^{2}$ and BINGHUA JIAO ${ }^{2}$ \\ ${ }^{1}$ Department of Biochemistry and Psychopharmacology, Shanghai Mental Health Center, \\ Shanghai Jiaotong University School of Medicine; ${ }^{2}$ College of Basic Medical Sciences, \\ The Second Military Medical University, Shanghai, P.R. China
}

Received September 18, 2013; Accepted April 3, 2014

DOI: $10.3892 / \mathrm{mmr} .2014 .2254$

\begin{abstract}
Tanshinone IIA (Tan IIA), one of the major active constituents of the medicinal herb Salvia miltiorrhiza, has been reported to possess neuroprotective effects against the pathological features of Alzheimer's disease (AD), but the molecular mechanism underlying this effect remains unclear. To examine the effect of Tan IIA on AD, as well as the underlying molecular mechanisms, in vivo animal experiments and in vitro molecular biology investigations were employed in the present study. Firstly, a rat model of AD was successfully established by direct injection of the amyloid beta protein $(\mathrm{A} \beta)$ and then these rats were administered an interventional treatment of Tan IIA. The learning and memory ability of rats was evaluated in four groups (Control, Sham, AD and Tan IIA) utilizing a Morris water maze test. Quantitative (q)PCR was employed to detect the mRNA expression of inducible nitric oxide synthase (iNOS), matrix metalloproteinase-2 (MMP-2) and nuclear transcription factor kappa $(\mathrm{NF}-\kappa \mathrm{Bp} 65)$ in temporal lobe tissues and protein expression was determined with western blot analysis. In addition, association analyses between iNOS, MMP-2 and NF-кBp65 at a transcriptional and translational level were performed utilizing Spearman's correlation analysis. In the present study, the results revealed that rats in the AD group demonstrated significant disruptions in learning and memory ability, and the symptoms were evidently reduced by Tan IIA. Furthermore, the upregulation of iNOS, MMP-2 and NF- $\mathrm{NBp} 65$ at a transcriptional and translational level in
\end{abstract}

Correspondence to: Dr Ping Jiang, Department of Biochemistry and Psychopharmacology, Shanghai Mental Health Center, Shanghai Jiaotong University School of Medicine, 600 Wan Ping Nan Road, Shanghai 200030, P.R. China

E-mail: jiangping021@126.com

Key words: Alzheimer's disease, Tanshinone IIA, iNOS, MMP-2, $\mathrm{NF}-\kappa \mathrm{Bp} 65$, mechanism
AD rats was distinctly inhibited by Tan IIA. Therefore, it was concluded that iNOS, MMP-2 and NF- $\kappa$ Bp65 are involved in $\mathrm{AD}$ development, and Tan IIA may reduce AD risk by inhibiting transcription and translation of these genes. Furthermore, the positive correlation of iNOS and MMP-2 with NF- $\kappa \mathrm{Bp} 65$, respectively, provides evidence supporting the hypothesis that Tan IIA reduces AD risk by inhibiting iNOS and MMP-2 at a transcriptional and translational level through the NF- $\kappa \mathrm{B}$ pathway. In summary, Tan IIA is an effective neuroprotective agent for AD therapy, and iNOS, MMP-2 and NF- $\mathrm{Bp} 65$ may be the potential molecular targets for manipulating this effect therapeutically.

\section{Introduction}

Alzheimer's disease (AD) is a regressive disease of the central nervous system (CNS), characterized by progressive cognitive dysfunction, including memory decline, with the pathological features of senile plaques deposition, neurofibrillary tangles (NFT) and neurodegeneration of the brain. These pathological hallmarks occur predominantly in the temporal and frontal lobes of the cerebral cortex, hippocampus and the basal forebrain. Temporal lobe atrophy of AD patients is more severe than that of healthy individuals. This is consistent with the clinical features of AD patients, which include a decrease in intelligence and the decline of short-term memory in the early stages. Therefore, studies investigating neurodegeneration within the temporal lobe will further elucidate the pathogenic mechanisms behind AD $(1,2)$.

Tanshinone IIA (Tan IIA), is an abietane-type diterpene quinone, extracted from the traditional Chinese herbal medicine Salvia miltiorrhiza (Danshen) which is a well-established medicine in the treatment of cardiovascular diseases (3). Currently, emerging in vivo evidence is reporting that Tan IIA has neuroprotective effects on the cholinergic system, and appears to result in the improvements in the pathological changes caused by AD, but the underlying mechanisms of these effects is largely unclear (4). Previously, two genes, inducible nitric oxide synthase (iNOS) and MMP-2, have been reported 
as crucial in the development and pathogenesis of $\mathrm{AD}(5,6)$. As a result, we hypothesized that Tan IIA may reduce AD risk, through iNOS and MMP-2 signaling pathways.

iNOS is an important enzyme that mediates inflammatory processes. iNOS catalyzes the oxidative deamination of L-arginine to produce NO at high concentrations. NO is neuroprotective at low concentrations, but higher concentrations are potently neurotoxic to brain cells (7). In AD patients, studies have demonstrated that the number of iNOS-positive neurons is significantly enhanced in the brain, and is accompanied by abundant neuronal damage. This evidence suggested that iNOS may be associated with the pathogenesis of AD (8). Matrix metalloproteinase 2 (MMP-2) is a crucial zinc-dependent protease, involved in remodeling the extracellular matrix and modifying cell-cell and cell-matrix interactions (9). MMP-2 participates in the development of AD by regulating ionic concentrations, which mediate the balance of amyloid beta protein $(\mathrm{A} \beta)$ metabolism, therefore changes in MMP-2 levels are considered as a potential diagnostic biomarker of AD.

iNOS and MMPs were activated by the $N F-\kappa B$ pathway in the progression of cerebral aneurysm (10). However, few studies have demonstrated the activation of iNOS and MMP-2 by NF- $\mathrm{BB}$ in AD development. Nuclear transcription factor kappa B $(\mathrm{NF}-\kappa \mathrm{B})$ participates in multiple inflammatory immunoreactions. Furthermore, it has a regulatory role in the transcriptional and modulatory processes of numerous inflammatory mediator genes, including adhesion molecules, cytokines, inflammatory mediators and proteases. Accumulated evidence suggests that $\mathrm{NF}-\kappa \mathrm{B}$ is correlated with $\mathrm{AD}$ pathogenesis, and involved in the mechanism of $\mathrm{A} \beta$ injury (11), therefore, $\mathrm{NF}-\kappa \mathrm{B}$ may be the upstream activator that regulates iNOS and MMP-2, and further contributes to $\mathrm{AD}$ development induced by $\mathrm{A} \beta$.

In the present study, we established AD model rats with the method of direct $A \beta$ injection, then administered a interventional treatment with Tan IIA. Following this, the learning and memory ability of rats was examined in each group, and the mRNA and protein expression of iNOS, MMP-2 and NF- $\kappa$ Bp65 in temporal lobes was detected, respectively. The aim of the present study was to investigate the protective effect of Tan IIA on the learning and memory ability of AD rats, to confirm the potential correlation between the three genes and AD pathogenesis at a transcriptional and translational level, as well as to provide evidence for our hypothesis that Tan IIA may reduce AD risk by inhibiting iNOS, MMP-2 expression at a transcriptional and translational level, through the $\mathrm{NF}-\kappa \mathrm{B}$ pathway.

\section{Materials and methods}

Reagents. All antibodies used in this study were purchased from Cayman Chemical (Ann Arbor, MI, USA), including anti-iNOS, anti-MMP-2, anti-NF- $\mathrm{Bp} 65$, goat polyclonal and anti-actin. One-step Extract RNA kit (TRIzol reagent kit) was purchased from Promega, Inc. (Madison, WI, USA). PrimeScript reverse-transcript reagent kit and SYBR Premix Ex Taq ${ }^{\mathrm{TM}}$ enzyme were obtained from Takara Bio, Inc. (Shiga, Japan); A $\beta$, Tan IIA (degree of purity, >99\%) and other biochemistry reagents were purchased from Sigma-Aldrich
(St. Louis, MO, USA). Finally, the determination of protein content kit was from Bio-Rad (Hercules, CA, USA).

Animals. The adult male Sprague-Dawley rats ( $\mathrm{n}=60$; weighing, 200-250 g; age range, 8-12 weeks) used in the present study were obtained from the Laboratory Animal Center of the Second Military Medical University (Shanghai, China). All rats were housed in a climate-controlled room (temperature, $22-24^{\circ} \mathrm{C}$; humidity, $65-80 \%$ ) with free access to food and water. The present study was conducted following internationally recognized guidelines on animal welfare, as well as the principles issued by the State Scientific and Technological Commission (Beijing, China).

AD model establishment. AD model rats were established with the method of direct $A \beta$ injection as previously described (12). Each rat received general anaesthesia with intraperitoneal injection of $100 \mathrm{~g} / \mathrm{l}$ chloral hydrate $(0.35 \mathrm{~g} / \mathrm{kg})$, which was then fixed by a stereotaxic instrument on top of the head. The rat's anterior fontanelle was exposed following skin disinfection and incision on the top of the head. With the use of a stereotaxically technique and rat brain map, a $1 \mathrm{~mm}$ diameter hole was produced in the skull $(3.0 \mathrm{~mm}$ behind bregma, $2.0 \mathrm{~mm}$ to the right of the center line). A microsyringe was vertically inserted to administer a $10 \mu \mathrm{l}$ injection of $\mathrm{A} \beta$ into the lateral ventricle ( $2.8 \mathrm{~mm}$ vertical from the brain surface). The needle remained in that position for $>5$ min following injection to ensure adequate diffusion of $\mathrm{A} \beta$, after which it was slowly withdrawn. Rats in AD and Tan IIA groups were injected with $10 \mu \mathrm{A} \beta$ and the sham-operation group with $10 \mu 1$ normal saline instead. All procedures were performed with the strict aseptic manipulation.

Rats in groups. The rats $(\mathrm{n}=60)$ were randomly assigned to four groups: normal group (Control), sham-operation group (Sham), AD group (AD) and Tan IIA group (Tan IIA), with 15 rats in each group. After the AD model was established (24 h), rats in the Tan IIA group were intragastrically administered with $50 \mathrm{mg} / \mathrm{kg} /$ day Tan IIA (melted in corn oil) for 15 days. The rats in the other three groups were fed with $50 \mathrm{mg} / \mathrm{kg} /$ day corn oil for 15 days. All the rats were subjected to the Morris water maze test and their behavioral indexes were recorded once daily from day 11-15 following the induction of AD. Following the test on the 15th day, all the rats were sacrificed, and their temporal lobes were extracted and placed onto ice and washed by icy normal saline. Half of the temporal lobes were used in the western blot analysis and the other in quantitative (q)PCR.

Morris water maze test. The Morris water maze was performed following the commonly used procedure as described previously (13). All of the rats were subject to the test once daily from day 11 to day 15 following the induction of experimental AD. For the training, the rats were placed into water at one of four quadrants around the pool's perimeter, facing the wall. Once the rat located the platform (escape latency; EL) within $60 \mathrm{sec}$, the training was terminated and EL was recorded as real time. Otherwise, the rat was guided to the platform and allowed to remain for $20 \mathrm{sec}$ and EL was $1 \mathrm{~min}$. 
Table I. Primers of iNOS, MMP-2, NF- $\kappa$ Bp65 and $\beta$-actin.

\begin{tabular}{ll}
\hline Gene & \multicolumn{1}{c}{ Primer } \\
\hline iNOS & F: CTC ACT GTG GCT GTG GTC ACC TA \\
& R: GGG TCT TCG GGC TTC AGG TTA \\
MMP-2 & F: GAT CCG TGG TGA GAT CTT CTT C \\
& R: AGA ACA CAG CCT TCT CTT CCT G \\
NF-кBp65 & F: AGG ACC CAA GCA CCT TCT TT \\
& R: GGG ATT TTG TCG TTG CTT GT \\
$\beta$-actin & F: TGA CAG GTG CAG AAG GAG A \\
& R: TAG AGC CAC CAA TCC ACA CA
\end{tabular}

iNOS, inducible nitric oxide synthase; MMP-2, matrix metalloproteinase-2; NF- $\kappa \mathrm{B}$, nuclear transcription factor- $\kappa \mathrm{B}$.

$q P C R$. The expression of genes was confirmed by qPCR. Primers were designed and synthesized by Takara Bio, Inc. Total RNA was isolated from iced temporal lobe tissues using RNAiso reagent. First strand cDNA was synthesized from $0.5 \mu \mathrm{g}$ of total cellular RNA with random hexamers with the ExScript ${ }^{\mathrm{TM}}$ RT Reagents kit. qPCR cycles were conducted for amplification of iNOS, MMP-2, NF- $\kappa$ Bp65 and $\beta$-actin cDNA. The primer sequences are listed in Table I. Fluorescent dye used in qPCR reactions was SYBR Premix Ex $\mathrm{Taq}^{\mathrm{TM}}$. All reactions were performed in triplicate with the TaqMan EZ (reverse transcriptase) RT-PCR core kit in an ABI-7000 machine (Ambion, Applied Biosystems Foster City, CA, USA) according to the manufacturer's instructions. PCR was performed in a $25 \mu 1$ solution containing $400 \mathrm{nM}$ of the primer, $200 \mathrm{nM}$ probe, $300 \mu \mathrm{M}$ each of deoxynucleotide triphosphate (dNTP), $3.0 \mathrm{mM}$ manganese acetate, $2.5 \mathrm{U}$ DNA polymerase and the PCR buffer. The threshold cycle changes $(\Delta \mathrm{Ct})$ denote the difference in $\mathrm{Ct}$ of iNOS, MMP-2 or NF- $\kappa$ Bp65 from the $\mathrm{Ct}$ level of $\beta$-actin within the sample. Following incubation for $15 \mathrm{~min}$ at $42^{\circ} \mathrm{C}$, the PCR cycling program was set for one cycle of pre-denaturation at $95^{\circ} \mathrm{C}$ for $60 \mathrm{sec}$ and then 40 cycles at $95^{\circ} \mathrm{C}$ for $30 \mathrm{sec}, 95^{\circ} \mathrm{C}$ for $5 \mathrm{sec}$, $60^{\circ} \mathrm{C}$ plate reading for $30 \mathrm{sec}$, melting curve from $55-95^{\circ} \mathrm{C}$ read every $0.2^{\circ} \mathrm{C}$, holding for $1 \mathrm{sec}$ between reads. The relative copies of iNOS, MMP-2 and NF- $\kappa$ Bp65 mRNA on the internal control $\beta$-actin are in arbitrary units.

Western blot analysis. Temporal lobe samples were homogenized in an ice-cold lysis buffer ( $\mathrm{pH} 7.5)$ containing $20 \mathrm{mM}$ Tris, $150 \mathrm{mM} \mathrm{NaCl}, 10 \mathrm{mM} \mathrm{MgCl}{ }_{2}, 1.0 \mathrm{mM}$ EDTA, $1.0 \mathrm{mM}$ EGTA, $1 \%$ Triton $\mathrm{X}-100$ and a mixture of protease inhibitors. The homogenate was centrifuged at $15,000 \times \mathrm{g}$ at $4^{\circ} \mathrm{C}$ for $30 \mathrm{~min}$ and the supernatant was stored at $-80^{\circ} \mathrm{C}$ for the western blot analysis. Briefly, samples were incubated with a sodium dodecyl sulfate-polyacrylamide gel electrophoresis (SDS-PAGE) sample buffer for $5 \mathrm{~min}$ at $95^{\circ} \mathrm{C}$. Each sample (40 $\mu \mathrm{g} /$ lane) was then subjected to SDS-PAGE and the separated proteins were transferred for $60 \mathrm{~min}$ to a PVDF membrane (GE Healthcare, Buckinghamshire, UK). The membrane was first incubated with a blocking solution containing 0.3-2\% skimmed milk, $25 \mathrm{mM}$ Tris, $150 \mathrm{mM}$

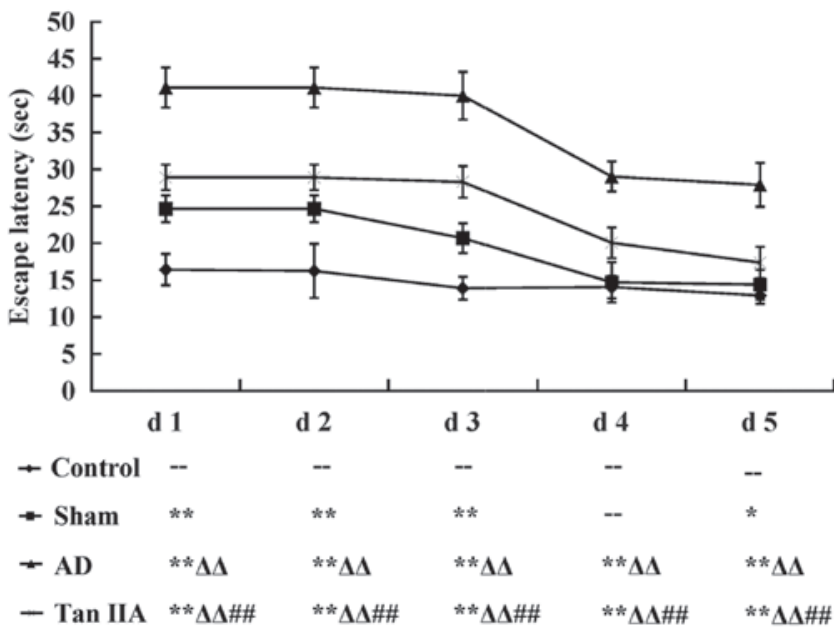

Figure 1. Tan IIA improves the learning and memory ability of AD rats. Rats in the AD group presented learning and memory dysfunction, as determined by the Morris water maze test, demonstrating significantly increased EL, compared with the Control and Sham groups ( $\mathrm{P}<0.01$, respectively). Tan IIA evidently relieved the cognitive dysfunction, as revealed by the shortened EL compared with the AD group. ${ }^{-} \mathrm{P}>0.05$ vs. Control group; ${ }^{*} \mathrm{P}<0.05$ vs. Control group; ${ }^{* *} \mathrm{P}<0.01$ vs. Control group; ${ }^{\Delta} \mathrm{P}<0.05$ vs. Sham group; ${ }^{\Delta \Delta} \mathrm{P}<0.05$ vs. Sham group; ${ }^{\#} \mathrm{P}<0.05$ vs. AD group; ${ }^{\# \#} \mathrm{P}<0.01$ vs. AD group. $\mathrm{AD}, \mathrm{Alzheimer}$ 's disease; Tan IIA, tanshinone IIA; EL, escape latency.

$\mathrm{NaCl}$ and $0.1 \%$ Tween-20 (pH 7.5) for $60 \mathrm{~min}$, then with the corresponding primary antibodies overnight $\left(4^{\circ} \mathrm{C}\right)$. The primary antibodies were a rabbit polyclonal antibody against iNOS (1:500), a goat polyclonal antibody against MMP-2 (1:400), a goat polyclonal antibody against NF-кBp65 $(1: 400)$ and mouse monoclonal antibodies against $\beta$-actin $(1: 1,000)$. Following this, the membranes were placed into the secondary antibody solution and incubated for $60 \mathrm{~min}$. The corresponding secondary antibody was a goat anti-rabbit IgG-HRP conjugate $(1: 2,000)$ for iNOS, a donkey anti-goat IgG-HRP conjugate $(1: 2,000)$ for MMP-2 and NF- $\kappa$ Bp65 and a sheep anti-mouse IgG-HRP conjugate $(1: 2,000)$ for $\beta$-actin. Final detection was performed with the enhanced chemiluminescence methodology (Amersham ECL western blotting detection reagents and analysis system; GE Healthcare) using a lumino imaging analyzer (LAS-3000; FUJIFILM, Tokyo, Japan). To normalize for protein loading, chemiluminescence of the bands in each lane was standardized to the intensity of the $\beta$-actin band in the same lane.

Statistical analysis. Data are presented as the mean \pm SD and one-way analysis of variance (ANOVA) was used to determine the effect of AD or Tan IIA. Association analysis among iNOS, MMP-2, NF- $\mathrm{Bp} 65$ expression at mRNA and protein level was performed by Spearman's correlation analysis. A value of $\mathrm{P}<0.05$ was considered to indicate a statistically significant difference.

\section{Results}

Tan IIA improves the learning and memory ability of AD rats. The Morris water maze test was conducted to investigate the learning and memory ability of each rat in the four groups. Mean EL was considered as the quantitative indicator of the behavioral observations representative of 


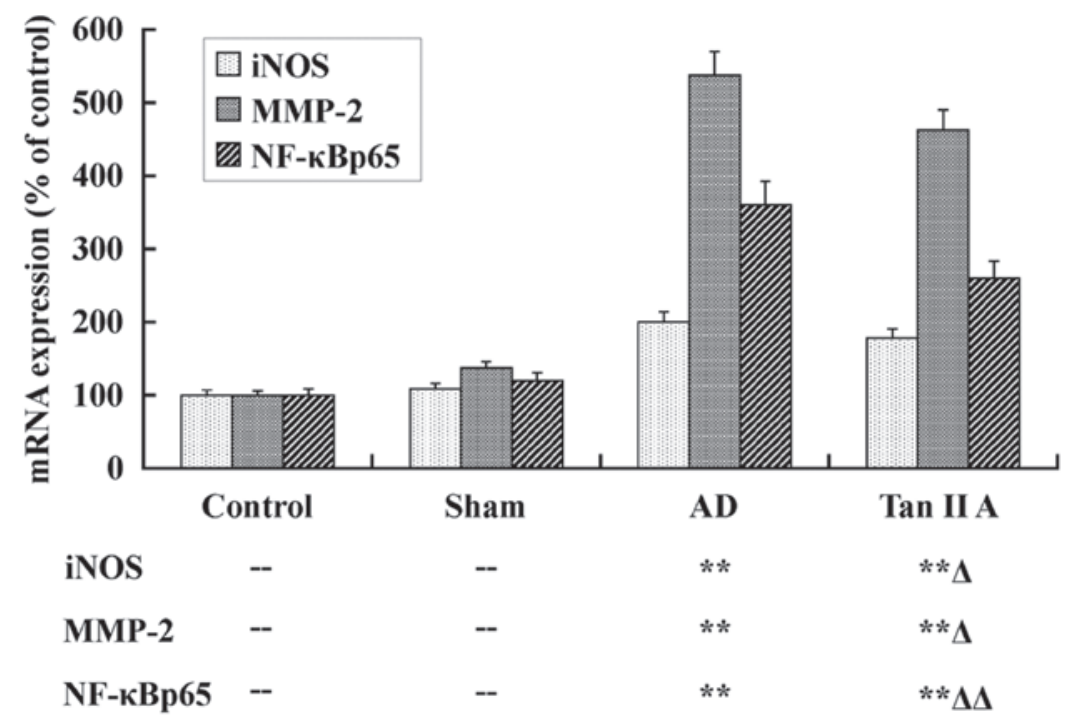

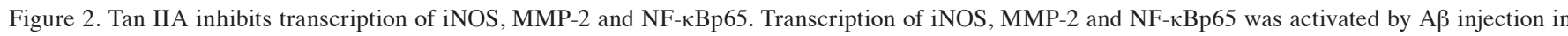
the AD group with higher mRNA expression than the Control and Sham groups, yet the upregulated mRNA expression of the three genes was distinctively inhibited by Tan IIA ( $\mathrm{P}<0.01$, respectively) in the Tan IIA group. ${ }^{-} \mathrm{P}>0.05$ vs. Control group; ${ }^{*} \mathrm{P}<0.05$ vs. Sham group; ${ }^{* *} \mathrm{P}<0.01$ vs. Sham group; ${ }^{\Delta} \mathrm{P}<0.05$ vs. AD group; ${ }^{\Delta \Delta} \mathrm{P}<0.05$ vs. AD group. AD, Alzheimer's disease; Tan IIA, Tanshinone IIA; iNOS, inducible nitric oxide synthase; MMP-2, matrix metalloproteinase-2; $\mathrm{NF}-\mathrm{\kappa B}$, nuclear transcription factor- $\mathrm{kB}$.

A

iNOS

\section{Control}

Sham

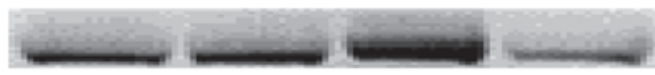

MMP-2

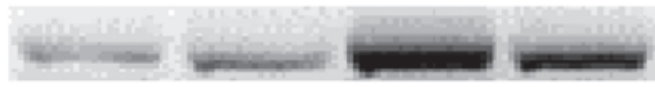

NF-кBp65

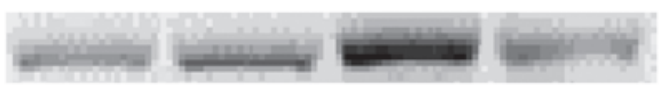

$\beta$-actin

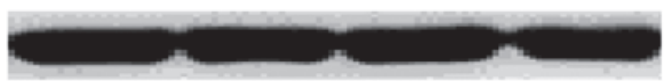

B

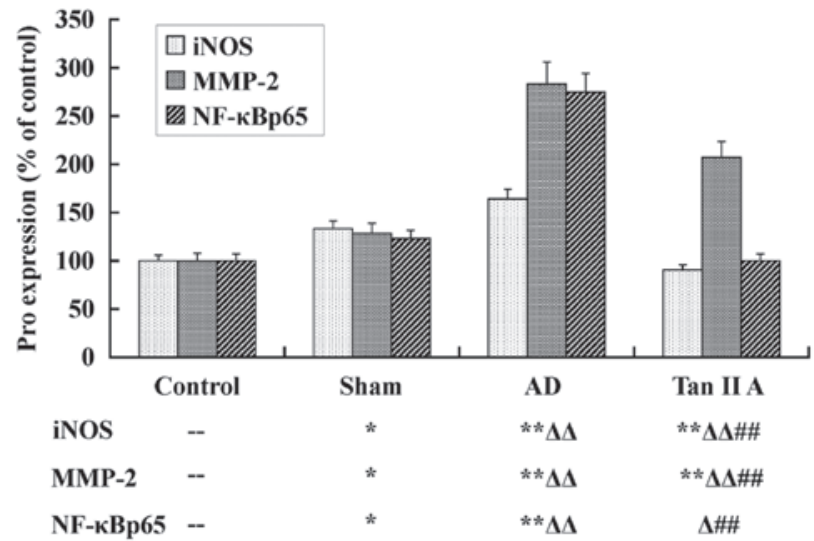

Figure 3. Tan IIA inhibits the translation of iNOS, MMP-2 and NF- $\mathrm{kBp} 65$. The results of the western blot analysis are presented; (A) the image produced by western blot assay and (B) the column diagram calculated and transformed from the gamma value of each stripe. A $\beta$ injection induced the upregulation of iNOS, MMP-2 and NF- $\mathrm{kBp} 65$ proteins in the AD group, and Tan IIA significantly inhibited the enhanced translation of the three proteins $(\mathrm{P}<0.01$, respectively) in the Tan IIA group. $-\mathrm{P}>0.05$ vs. Control group; ${ }^{*} \mathrm{P}<0.05$ vs. Control group; ${ }^{* *} \mathrm{P}<0.01$ vs. Control group; ${ }^{\Delta} \mathrm{P}<0.05$ vs. Sham group; ${ }^{\Delta \Delta} \mathrm{P}<0.05$ vs. Sham group; ${ }^{\#} \mathrm{P}<0.05$ vs. AD group; ${ }^{\# \#} \mathrm{P}<0.01$ vs. AD group. $\mathrm{A} \beta$, amyloid $\beta$; $\mathrm{AD}$, Alzheimer's disease; Tan IIA, Tanshinone IIA; iNOS, inducible nitric oxide synthase;

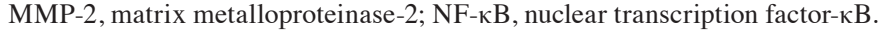

cognitive function. Data was recorded as the mean $\pm \mathrm{SD}$ and then transformed to the line chart illustrated in Fig. 1. Each group demonstrated a progressive decline in EL from day 1-5 $(\mathrm{P}<0.05)$. Compared with the Control group, EL was significantly longer in the Sham group from day 1-5 except for day $4(\mathrm{P}>0.05)$. However, the AD group had a steeper increase in EL than the Sham group from day 1-5 $(\mathrm{P}<0.01$, respectively). The results indicated that the AD model was successfully established in rats by the direct injection of $\mathrm{A} \beta$ and therefore were used in the experiments. When the comparison was performed between AD and Tan IIA groups, it was identified that EL reduced markedly in the Tan IIA group from day 1-5 ( $\mathrm{P}<0.01$ respectively), which suggested that Tan IIA improved the cognitive dysfunction in learning and memory exhibited by the $\mathrm{AD}$ rats.

Tan IIA inhibits the mRNA expression of iNOS, MMP-2 and $N F-\kappa B p 65$. The mRNA expression of iNOS, MMP-2

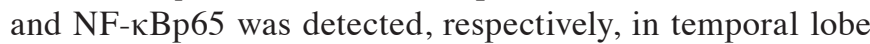
tissues of the four groups utilizing qPCR, to elucidate the protective effect of Tan IIA at a transcriptional level. The results are presented as a column diagram in Fig. 2. There was no significant difference in expression of all three genes between the Sham and Control groups $(\mathrm{P}>0.05)$. However, in the AD group, the mRNA expression of iNOS, MMP-2 and NF- $\kappa B p 65$ were evidently upregulated $(\mathrm{P}<0.01$, respectively). 
Table II. Spearman's correlation analysis on the mRNA expression of iNOS, MMP-2 and NF-kBp65.

\begin{tabular}{lccl}
\hline Index & iNOS & MMP-2 & NF-kBp65 \\
\hline iNOS & - & $\mathrm{r}_{\mathrm{s}}=0.998^{* *}$ & $\mathrm{r}_{\mathrm{s}}=0.992^{* *}$ \\
MMP-2 & $\mathrm{r}_{\mathrm{s}}=0.998^{* *}$ & - & $\mathrm{r}_{\mathrm{s}}=0.985^{*}$ \\
NF-kBp65 & $\mathrm{r}_{\mathrm{s}}=0.992^{* *}$ & $\mathrm{r}_{\mathrm{s}}=0.985^{*}$ & - \\
\hline
\end{tabular}

${ }^{*} \mathrm{P}<0.05 ;{ }^{* *} \mathrm{P}<0.01$. iNOS, inducible nitric oxide synthase; MMP-2, matrix metalloproteinase-2; NF- $\mathrm{B}$, nuclear transcription factor- $\mathrm{B}$.

Table III. Spearman's correlation analysis on protein expressions of iNOS, MMP-2 and NF-kBp65.

\begin{tabular}{|c|c|c|c|}
\hline Index & iNOS & MMP-2 & NF-кBp65 \\
\hline iNOS & - & $\mathrm{r}_{\mathrm{s}}=0.822$ & $\mathrm{r}_{\mathrm{s}}=0.974^{*}$ \\
\hline MMP-2 & $\mathrm{r}_{\mathrm{s}}=0.822$ & - & $\mathrm{r}_{\mathrm{s}}=0.911$ \\
\hline NF-кBp65 & $r_{s}=0.974^{*}$ & $r_{s}=0.911$ & - \\
\hline
\end{tabular}

${ }^{*} \mathrm{P}<0.05 ;{ }^{* *} \mathrm{P}<0.01$. iNOS, inducible nitric oxide synthase; MMP-2, matric metalloproteinase-2; NF- $\kappa \mathrm{B}$, nuclear transcription factor- $\kappa \mathrm{B}$.

These data demonstrated that the higher mRNA expression levels of iNOS, MMP-2 and NF- $\kappa$ Bp65 in temporal lobe tissues were associated with AD. Most importantly, in our further investigations, the mRNA expression of iNOS, MMP-2 and NF- $\kappa B p 65$ was distinctly reduced in the Tan IIA group compared with the $\mathrm{AD}$ group $(\mathrm{P}<0.05, \mathrm{P}<0.05$ and $\mathrm{P}<0.01$, respectively).

Tan IIA reduces the protein expression of iNOS, MMP-2 and $N F-\kappa B p 65$. Western blot analysis was utilized to determine the expression at a translational level. The protein expression of iNOS, MMP-2 and NF-kBp65 was compared among the four groups (Fig. 3). There was a significant upregulation in the protein expression of iNOS, MMP-2 and NF- $\mathrm{kBp} 65$ in the Sham group compared with the Control group $(\mathrm{P}<0.05$, respectively). The $\mathrm{AD}$ group had a highly significant increase in all three protein expression levels compared with the Sham group $(\mathrm{P}<0.01$, respectively). The findings suggested that the elevated protein expression of iNOS, MMP-2 and NF- $\mathrm{kBp} 65$ in the temporal lobe tissues was also associated with AD. Furthermore, the expression of the three proteins in the Tan IIA group reduced markedly compared with the AD group $(\mathrm{P}<0.01$, respectively), which indicated that Tan IIA reduced the protein expression of iNOS, MMP-2 and NF- $\kappa B p 65$ in the temporal lobe tissues of $\mathrm{AD}$ rats.

Association between iNOS, MMP-2 and NF- $\kappa B p 65$ at transcriptional and translational levels. Spearman's correlation analysis on the mRNA expression of iNOS, MMP-2 and NF- $\kappa$ Bp 65 suggested that any two of the three genes had an evident positive correlation, as summarized in Table II. The strongest correlation was identified between iNOS and MMP-2 with $\mathrm{r}_{\mathrm{s}}=0.998$ and $\mathrm{P}<0.01$. At a translation level, a significantly positive correlation was identified between iNOS and NF-kBp65 with $\mathrm{r}_{\mathrm{s}}=0.974$ and $\mathrm{P}<0.05$, as shown in Table III.

\section{Discussion}

Patients with AD consistently suffer from symptoms of cognitive impairment, with memory dysfunctions occurring at an early stage in the disease. Compared with the healthy brain, AD patients have significant atrophy of the temporal lobe, lower bleeding through part of temporal lobes. Studies that focus on neurodegeneration in the temporal lobe will certainly contribute to the elucidation of AD pathogenesis. A number of animal experiments have demonstrated that Tan IIA may protect the cholinergic system in the brains of $\mathrm{AD}$ rats and improve the pathological alterations induced by $\mathrm{AD}$, but the mechanism underlying this effect was largely unclear. In the present study, the protective effect of Tan IIA on the learning and memory ability of AD rats was investigated, by means of the Morris water maze behavioral test. We further analyzed the effect of Tan IIA on the expression of the three genes closely associated with $\mathrm{AD}$ in the temporal lobe tissues of $\mathrm{AD}$ rats, and attempted to clarify the protective mechanism at a transcriptional and translational level.

$\mathrm{An}$ in vivo $\mathrm{AD}$ model was established in rats by direct $\mathrm{A} \beta$ protein injection. The $\mathrm{AD}$ rats demonstrated an evident dysfunction in learning and memory in the Morris water maze test, while, Tan IIA distinctly reduced this effect. Consequently, the results confirmed that Tan IIA had a protective effect on learning and memory ability of $\mathrm{AD}$ rats.

At a transcriptional and translational level, iNOS and MMP-2 in the temporal lobe tissues of AD rats was significantly upregulated, which was consistent with the results of our previous study in hippocampus of AD rats (14). From these findings, we make several conjectures. First, MMPs are one of the most important metalloenzymes, which are closely associated with $\mathrm{A} \beta$ metabolism. $\mathrm{A} \beta$ metabolism disorder in $\mathrm{AD}$ rats induces changes in the concentrations of metal irons, which further induces enhanced expression of MMP-2 in temporal lobes. Secondly, iNOS is highly correlated to neurotoxicity induced by $A \beta$ according to previous studies. $A \beta$ injection activates the NO/NOS pathway, in which higher concentrations of NO produced by iNOS mediate delayed neuronal death following damage. Then the loss of neurons in brain causes cognitive decline, particularly dysfunctions of learning and memory $(12,15)$. In the present study, these data demonstrated an enhanced expression of iNOS in temporal lobe tissues of $\mathrm{AD}$ rats, accompanied with the dysfunction of learning and memory, providing evidence for the above conjectures. Furthermore, the results that Tan IIA inhibited the expression of iNOS and MMP-2 at a transcriptional and translational level in temporal lobe tissues, confirmed the involvement of iNOS and MMP-2 pathways in AD pathogenesis and development.

In order to further elucidate the mechanisms involved, we detected the alterations of the potential upstream molecular pathway, NF- $\kappa \mathrm{Bp} 65$. As the most general subtype of NF- $\kappa \mathrm{B}$ protein family, NF- $\kappa \mathrm{Bp} 65$ is expressed in almost all cells and not only participates in normal immune and inflammatory reactions, but is also important in the regulation of various genes which are involved in AD pathology (16). Furthermore, 
the p65 subtype may improve the transcriptional activation of target genes with the transcription-activating domain located in the C-terminal, or exert its effects through being coupled with other I $\kappa$ B members (17). Enhanced NF- $\kappa$ Bp65 activity has been identified in the brain tissue of AD patients, including in degenerating neurons and in neurons adjacent to senile plaques and glial cells. NF- $\mathrm{Bp} 65$ is highly associated with the pathogenesis of AD. Furthermore, as $\mathrm{NF}-\kappa \mathrm{B}$ in neurons may be activated by $A \beta$ deposition, the $N F-\kappa B$ pathway could be involved in $\mathrm{A} \beta$ damage mechanism in $\mathrm{AD}$ (18). In the present study, NF- $\kappa$ Bp65 were upregulated in temporal lobe tissues of $\mathrm{AD}$ rats at a transcriptional and translational level, which indicated that $\mathrm{NF}-\kappa \mathrm{Bp} 65$ is involved in $\mathrm{AD}$ development induced by $A \beta$ injection. In a similar manner, the inhibitory effects of Tan IIA on the mRNA and protein expression of $\mathrm{NF}-\kappa \mathrm{Bp} 65$ was accompanied by restrained AD risk and this further demonstrated that NF- $\kappa$ B pathway participated in $A D$ development in the AD rats. Furthermore, the results suggest that Tan IIA may reduce AD risk through the NF- $\kappa \mathrm{B}$ pathway.

In the present study, it was identified that iNOS, MMP-2 and $\mathrm{NF}-\kappa \mathrm{Bp} 65$ are involved in AD development, as well as the neuroprotective effects of Tan IIA on AD. To determine whether the activation of iNOS and MMP-2 in AD rats was induced by the NF- $\mathrm{NB}$ pathway, an association analysis among iNOS, MMP-2 and NF- $\kappa$ Bp65 was performed. As a result, a highly positive correlation between any two of the three genes was identified, as was a positive association between iNOS and the NF- $\mathrm{Bp} 65$ protein. According to several other studies, there is a $\kappa \mathrm{B}$ element in the promoter of iNOS and MMP-2, respectively, which provides the binding site for the signal molecules of the NF- $\kappa \mathrm{B}$ pathway (17). Consequently, we can infer that, the upregulation of mRNA and protein expression of NF- $\kappa$ Bp65 activates iNOS transcription and translation to produce high concentrations of NO, which may lead to the learning and memory dysfunction of $\mathrm{AD}$ rats. By contrast, the activated NF- $\kappa \mathrm{Bp} 65$ targets the $\kappa \mathrm{B}$ sequence of MMP-2 promoter to upregulate MMP-2 gene expression, which aggravates the neurological disorder in AD rats. Furthermore, Tan IIA inhibits the activation of NF- $\mathrm{BB}$ pathway, thus inhibiting the iNOS and MMP-2 signaling pathways to reduce the risk of AD stimulated by $A \beta$ injection.

Based on the findings of the present study, we conclude that iNOS, MMP-2 and NF- $\kappa$ Bp65 are involved in the development of $\mathrm{AD}$ induced by $\mathrm{A} \beta$ injection, and Tan IIA may reduce the risk of $\mathrm{AD}$ by inhibiting iNOS, MMP-2 and NF- $\kappa \mathrm{Bp} 65$ expression at the transcriptional and translational levels in temporal lobe tissues of AD rats. Additionally, these results provide further evidence for the hypothesis that Tan IIA may reduce AD risk by inhibiting iNOS and MMP-2 expression through the NF- $\mathrm{KB}$ pathway. However, further studies should be conducted to elucidate the mechanism underlying this effect in detail. Nevertheless, iNOS, MMP-2 and NF-кBp65 are potential targets in $\mathrm{AD}$ prophylaxis and treatment because they are not only accurate biomarkers that indicate the progression of $\mathrm{AD}$, but are also key targets against $\mathrm{A} \beta$ deposition $(19,20)$. Furthermore, we conclude Tan IIA is an effective neuroprotective agent for AD therapy, and iNOS, MMP-2 and $\mathrm{NF}-\kappa \mathrm{Bp} 65$ may be the potential targets molecular targets for manipulating this effect therapeutically.

\section{Acknowledgements}

The present study was supported by grants from Shanghai Health Bureau Scientific Research Project (no. 20114297) and Shanghai Jiaotong University School of Medicine Nature Science Fund Project (no. 11XJ21068).

\section{References}

1. Taly A, Corringer PJ, Guedin D, Lestage P and Changeux JP: Nicotinic receptors: allosteric transitions and therapeutic targets in the nervous system. Nat Rev Drug Discov 8: 733-750, 2009.

2. Beagley KW, Huston WM, Hansbro PM and Timms P: Chlamydial infection of immune cells: altered function and implications for disease. Crit Rev Immunol 29: 275-305, 2009.

3. Mei Z, Zhang F, Tao L, et al: Cryptotanshinone, a compound from Salvia miltiorrhiza modulates amyloid precursor protein metabolism and attenuates beta-amyloid deposition through upregulating alpha-secretase in vivo and in vitro. Neurosci Lett 452: 90-95, 2009.

4. Wang Q, Yu X, Patal K, Hu R, Chuang S, Zhang G and Zheng J: Tanshinones inhibit amyloid aggregation by amyloid- $\beta$ peptide, disaggregate amyloid fibrils, and protect cultured cells. ACS Chem Neurosci 4: 1004-1015, 2013

5. Malinski T: Nitric oxide and nitroxidative stress in Alzheimer's disease. J Alzheimers Dis 11: 207-218, 2007.

6. Durrant JD, de Oliveira CA and McCammon JA: Including receptor flexibility and induced fit effects into the design of MMP-2 inhibitors. J Mol Recognit 23: 173-182, 2010.

7. Steinert JR, Chernova T and Forsythe ID: Nitric oxide signaling in brain function, dysfunction, and dementia. Neuroscientist 16: 435-452, 2010

8. Wolk DA and Klunk W: Update on amyloid imaging: from healthy aging to Alzheimer's disease. Curr Neurol Neurosci Rep 9: 345-352, 2009.

9. Romi F, Helgeland G and Gilhus NE: Serum levels of matrix metalloproteinases: implications in clinical neurology. Eur Neurol 67: 121-128, 2012.

10. Aoki T, Kataoka H, Shimamura M, et al: NF-kappaB is a key mediator of cerebral aneurysm formation. Circulation 116: 2830-2840, 2007.

11. Zhang J, Zhen YF, Pu-Bu-Ci-Ren, et al: Salidroside attenuates beta amyloid-induced cognitive deficits via modulating oxidative stress and inflammatory mediators in rat hippocampus. Behav Brain Res 244: 70-81, 2013.

12. Limpeanchob N, Jaipan S, Rattanakaruna S, Phrompittayarat W and Ingkaninan K: Neuroprotective effect of Bacopa monnieri on beta-amyloid-induced cell death in primary cortical culture. J Ethnopharmacol 120: 112-117, 2008.

13. Nakashima Y, Ohsawa I, Konishi F, et al: Preventive effects of Chlorella on cognitive decline in age-dependent dementia model mice. Neurosci Lett 464: 193-198, 2009.

14. Jiang P, Chen M, Lv J, Chen C and Jiao BH: Effect of tanshinone II A on MMP-2 and iNOS expression and free radical release in hippocampus of rat Alzheimer's disease model. Academic Journal of Second Military Medical University 31: 380-384, 2010 (In Chinese).

15. Silveira LR, Pereira-Da-Silva L, Juel C and Hellsten Y: Formation of hydrogen peroxide and nitric oxide in rat skeletal muscle cells during contractions. Free Radic Biol Med 35: 455-464, 2003.

16. Ascolani A, Balestrieri E, Minutolo A, et al: Dysregulated $\mathrm{NF}-\kappa \mathrm{B}$ pathway in peripheral mononuclear cells of Alzheimer's disease patients. Curr Alzheimer Res 9: 128-137, 2012.

17. Nomura Y: NF-kappaB activation and IkappaB alpha dynamism involved in iNOS and chemokine induction in astroglial cells. Life Sci 68: 1695-1701, 2001.

18. Akhand AA, Du J, Liu W, et al: Redox-linked cell surface-oriented signaling for T-cell death. Antioxid Redox Signal 4:445-454, 2002.

19. Malinski T: Nitric oxide and nitroxidative stress in Alzheimer's disease. J Alzheimers Dis 11: 207-218, 2007.

20. Rosenberg GA: Matrix metalloproteinases and their multiple roles in neurodegenerative diseases. Lancet Neurol 8: 205-216, 2009. 